\title{
Displasias esqueléticas y factores de riesgo asociados. Descripción de 29 casos reportados en seis hospitales de Bogotá, Cali y Manizales
}

\author{
Fernando Rodríguez Guevara ${ }^{1}$, Lili Johana Rueda Jaime ${ }^{1}$, \\ Ignacio Manuel Zarante Montoya ${ }^{2}$
}

\section{Resumen}

Objetivo. Hacer un análisis de los posibles factores de riesgo a partir de una serie de casos de displasias esqueléticas que se han reportado en los últimos ocho años en algunas ciudades de Colombia.

Métodos. Se desarrolló un estudio de casos y controles en seis hospitales de tres ciudades (Cali, Manizales y Bogotá) que participan en el Estudio Colaborativo Latinoamericano de Malformaciones Congénitas. Para el análisis se incluyeron 29 casos y 85 controles.

Resultados. Respecto a las variables cuantitativas, se encontraron diferencias estadísticamente significativas entre ambos grupos para edad paterna, edad de gestación, número de gestaciones y peso al nacer. Para las variables cualitativas, los casos presentaron un riesgo mayor de tener un peso menor de $2.500 \mathrm{~g}$ al nacer y un bajo peso para la edad de gestación.

Conclusiones. Las displasias esqueléticas son una malformación poco frecuente en la población estudiada. En más de la mitad de los casos no se hace un diagnóstico específico. Esta malformación se asocia en esta muestra con avanzada edad paterna, bajo peso al nacer, edad de gestación y número de gestaciones.

Palabras clave: anomalías congénitas, desarrollo óseo, diagnóstico prenatal.

1 Médico(a) y cirujano(a), Facultad de Medicina, Pontificia Universidad Javeriana, Bogotá, D.C., Colombia.

2 Médico y cirujano, Facultad de Medicina, Pontificia Universidad Javeriana; maestría en Ciencias Biológicas con énfasis en Genética, Profesor Instituto de Genética Humana, Facultad de Medicina, Pontificia Universidad Javeriana, Bogotá, D.C., Colombia. 
Title

Skeletal dysplasia and associated risk factors Description of 29 cases reported in 6 hospitals from Bogotá, Cali and Manizales

\begin{abstract}
Objective: Our aim was to conduct an analysis of risk factors from a population with skeletal dysplasia notified in some cities of Colombia in the last eight years.
\end{abstract}

Methods: We conducted a case-control study in several Colombian hospitals participating in the Latin-American Collaborative Study of Congenital Malformations (ECLAMC, Spanish acronym). For the analysis 29 cases and 85 controls were included.

Results: In the quantitative variables we found statistical significance between cases and controls for paternal age, gestational age, number of gestations and birth weight. With regard to qualitative variables, the cases had a higher risk of birth weight less than $2500 \mathrm{~g}$ and low birth weight for the gestational age.

Conclusions: Skeletal dysplasias are a congenital malformation with a low frequency in the population studied and often lacking of a specific diagnostic. They are associated with advanced paternal age, low birth weight, gestational age and number of gestations.

Key words: congenital abnormalities, bone development, prenatal diagnosis.

\section{Introducción}

Las displasias esqueléticas, también conocidas como osteocondrodisplasias, son alteraciones que ocurren durante el desarrollo, crecimiento y mantenimiento de los huesos y cartílagos, que se manifiestan generalmente con baja talla desproporcionada y muchas veces con alteraciones en otros órganos y sistemas, aparte del esquelético[1-3]. Estas alteraciones tienen un rango de gravedad que varía desde la presencia de sutiles cambios morfológicos, hasta la muerte ocasionada principalmente por falla respiratoria[1,3].

Actualmente, la clasificación de alteraciones óseas constitucionales (Classification of Constitutional Disorders of Bone, CCDB) ha organizado en 33 grupos más de 200 displasias[4]. Muchas de estas alteraciones son el resultado de nuevas mutaciones dominantes y tienen un patrón de herencia autosómico recesivo; por ello, se ven frecuentemente en familias que no presentan antecedentes de esta enfermedad[5].

Si bien en los últimos años ha habido un progreso sustancial en la identificación y en el entendimiento de ciertos defectos genéticos responsables de la mayoría de displasias esqueléticas, se han realizado pocas investigaciones para tratar de dilucidar otras asociaciones no moleculares, como los factores de riesgo relacionados con la concepción y los agentes teratogénicos. Entre los pocos que se han llevado a cabo, se ha reportado la asociación de algunos tipos de displasias con la edad paterna avanzada y con el consumo de warfarina por la madre durante el embarazo[1].

Aunque cada displasia en particular tiene una baja prevalencia, todas juntas 
tienen una presentación relativamente común, que puede llegar hasta 7,6 casos por cada 10.000 nacimientos[6]. En Colombia, la frecuencia reportada es sólo de 2,46 casos por cada 10.000 nacimientos, cifra que podría indicar un posible subregistro de estas enfermedades[7]. De más de 200 displasias descritas, aproximadamente la mitad resultan letales y causan 9 de cada 1.000 muertes perinatales[1]. Debido a esto, el diagnóstico prenatal representa un importante eslabón en el abordaje multidisciplinario del paciente, el cual debe ser instaurado tempranamente para tratar de reducir la morbilidad, la mortalidad y las secuelas. Luego del nacimiento, es imprescindible confirmar el diagnóstico a la luz de los hallazgos clínicos, radiológicos y moleculares, lo cual a su vez permite clasificar correctamente el tipo de displasia, para brindar a los padres una correcta asesoría sobre el pronóstico clínico del paciente, así como del riesgo de recurrencia de la enfermedad en futuros embarazos[5].

El propósito de nuestro estudio fue analizar los posibles factores de riesgo de 29 casos de displasias esqueléticas que se han presentado en los últimos ocho años en algunas ciudades de Colombia mediante un sistema de vigilancia de malformaciones congénitas.

\section{Materiales y métodos}

Se trata de un estudio de casos y controles, en el que se incluyeron 29 casos de pacientes con diagnóstico de algún tipo de displasia esquelética y 85 controles (relación: 1:2,93). La información fue recolectada de la base de datos del Estudio Colaborativo Latinoamericano de Malformaciones Congénitas (ECLAMC), el cual es un programa de investigación clínica y epidemiológica de malformaciones congénitas mediante la vigilancia de los nacimientos en diferentes hospitales de Latinoamérica, bajo la metodología de casos y controles, y del cual el Instituto de Genética Humana es miembro activo. Los controles fueron el siguiente nacimiento sin malformación, en orden estrictamente cronológico, del mismo sexo que el del caso, de acuerdo con su clasificación preliminar. En ambos se solicitó el consentimiento informado para ingresar al proyecto.

Para este estudio se revisaron las fichas epidemiológicas de todos los nacimientos con malformaciones congénitas ocurridos entre 2001 y 2009 en cuatro hospitales de Bogotá, un hospital de Cali y un hospital de Manizales (tabla 1). Se incluyeron todos los casos con diagnóstico de displasia esquelética y se excluyeron aquellos con alteraciones de un proceso de desarrollo que en su origen fue normal. 
Tabla 1

Características demográficas de los hospitales

\begin{tabular}{lcccc}
\hline Hospital & Ciudad & $\begin{array}{c}\text { Nivel de } \\
\text { atención }\end{array}$ & $\begin{array}{c}\text { Régimen } \\
\text { principal }\end{array}$ & $\begin{array}{c}\text { Promedio de } \\
\text { partos al mes }\end{array}$ \\
\hline Hospital Universitario San Ignacio & Bogotá & IV & Contributivo & 280 \\
Fundación Emanuel & Bogotá & III & Contributivo & 300 \\
Clínica David Restrepo & Bogotá & IV & Contributivo & 250 \\
Hospital Simón Bolívar & Bogotá & III & Subsidiado & 120 \\
Hospital Universitario del Valle & Cali & IV & Subsidiado & 550 \\
Hospital Universitario de Caldas & Manizales & III & Subsidiado & 250 \\
\hline
\end{tabular}

Posteriormente, se hizo el análisis estadístico de las siguientes variables, para establecer asociaciones con las displasias esqueléticas: edad materna, edad paterna, edad de gestación, peso al nacer, número de gestaciones, número de ecografías prenatales, número de controles prenatales, presencia de síntomas durante el embarazo, enfermedades agudas o crónicas de la madre, metrorragia, consumo materno de tabaco, alcohol y sustancias psicoactivas, consumo de medicamentos y de vitaminas durante el embarazo e inmunoprofilaxis.

Las variables cuantitativas fueron analizadas utilizando la prueba $\mathrm{t}$ de Student, con un intervalo de confianza de 95\%, con Microsoft Excel ${ }^{\circledR}$, 2010. Las variables cualitativas se analizaron utilizando odds ratio (OR) con un intervalo de confianza de $95 \%$, con Epidat $^{\circledR}$, versión 3.1.

\section{Resultados}

Se encontraron 30 casos con diagnóstico de displasia esquelética; sin embargo, uno fue excluido por tratarse de un paciente con bridas amnióticas, por lo que finalmente se analizaron 29 casos y 85 controles de recién nacidos sanos, y se obtuvo una relación caso-control de $1: 2,93$. Estos casos y controles fueron obtenidos de los nacimientos reportados desde 2001 hasta 2009, en los hospitales de Bogotá, Cali y Manizales (tabla 2). De los 29 casos, 15 correspondían a displasias esqueléticas sin clasificar $(51,7 \%)$ y los 14 restantes $(48,3 \%)$, a displasias esqueléticas específicas (tabla 3).

Tabla 2

Distribución de frecuencia por ciudades de los casos

\begin{tabular}{lrc}
\hline Ciudad & $\mathrm{n}$ & $\%$ \\
\hline Cali & 16 & 55,2 \\
Bogotá & 9 & 31,0 \\
Manizales & 4 & 13,8 \\
\hline Total & 29 & \\
\hline
\end{tabular}


Tabla 3

Distribución de frecuencia por tipos de displasias esqueléticas incluidas en el análisis

\begin{tabular}{lccc}
\hline Diagnóstico & $\mathrm{n}$ & $\%$ & CIE-10 \\
\hline $\begin{array}{l}\text { Displasia esquelética sin clasificar } \\
\text { (otras osteocondrodisplasias) }\end{array}$ & 15 & 51,7 & Q78 \\
$\begin{array}{l}\text { Acondroplasia } \\
\text { Displasia tanatofórica }\end{array}$ & 4 & 13,8 & Q77.4 \\
$\begin{array}{l}\text { Defectos de reducción en miembros inferiores } \\
\text { (agenesia, atrofias o acortamientos) }\end{array}$ & 4 & 13,8 & Q77.1 \\
$\begin{array}{l}\text { Displasia campomélica } \\
\text { Osteogénesis imperfecta }\end{array}$ & 1 & 3,4 & Q78 \\
\hline Total & 1 & 3,4 & Q78 \\
\hline
\end{tabular}

Respecto a las variables cuantitativas, se encontraron diferencias estadísticamente significativas entre ambos grupos para edad paterna, edad de gestación, número de gestaciones y peso al nacer. No se encontraron diferencias significativas al comparar edad materna, número de controles prenatales y número de ecografías prenatales (tabla 4).

Tabla 4

Comparación de variables cuantitativas entre casos y controles

\begin{tabular}{lrrrrr}
\hline Variables & \multicolumn{2}{c}{ Casos } & \multicolumn{2}{c}{ Controles } & \multirow{2}{*}{$P$} \\
\cline { 2 - 5 } & Media & \multicolumn{1}{c}{ DE } & Media & DE & \\
\hline Edad paterna (años) & 34,55 & 9,42 & 27,55 & 7,73 & $\mathrm{p}<0,05$ \\
Edad de gestación (semanas) & 34,89 & 5,45 & 38,00 & 2,17 & $\mathrm{p}<0,05$ \\
Número de gestaciones & 2,48 & 1,52 & 1,65 & 0,94 & $\mathrm{p}<0,05$ \\
Peso al nacer (g) & $2.144,24$ & 844,00 & $2.959,75$ & 580,32 & $\mathrm{p}<0,05$ \\
Edad materna (años) & 26,31 & 5,98 & 28,83 & 6,92 & $\mathrm{p}>0,05$ \\
Número de controles prenatales & 5,34 & 3,33 & 5,10 & 3,03 & $\mathrm{p}>0,05$ \\
Número de ecografías prenatales & 2,82 & 1,69 & 2,36 & 1,81 & $\mathrm{p}>0,05$ \\
\hline
\end{tabular}

DE: desviación estándar 
En cuanto a las variables cualitativas, respecto a los controles, los casos presentaron un riesgo mayor de tener un peso menor de $2.500 \mathrm{~g}$ al nacer $(\mathrm{OR}=7,3$; $\left.\mathrm{IC}_{95 \%}, 2,99-19,44\right)$ y un bajo peso para la edad de gestación $\left(\mathrm{OR}=4,5 ; \mathrm{IC}_{95 \%}, 1,45\right.$ 13,99). No se encontraron diferencias significativas en el resto de variables analizadas (tabla 5).

\section{Tabla 5}

\section{Comparación de variables cualitativas entre casos y controles}

\begin{tabular}{|c|c|c|c|c|c|}
\hline \multirow[t]{2}{*}{ Variables } & \multicolumn{2}{|c|}{ Casos } & \multicolumn{2}{|c|}{ Controles } & \multirow[t]{2}{*}{ OR (IC 95\%) } \\
\hline & Sí & No & Sí & No & \\
\hline Edad paterna mayor a 45 años & 5 & 24 & 3 & 82 & $5,69(1,38-23,18)^{*}$ \\
\hline Edad paterna mayor a 40 años & 10 & 19 & 9 & 76 & $5,69(1,26-25,56)^{*}$ \\
\hline Peso $<2500 \mathrm{~g}$ al nacer & 18 & 11 & 15 & 70 & $7,3(2,99-19,44)^{*}$ \\
\hline Pequeño para la edad gestacional & 8 & 19 & 7 & 78 & $4,5(1,45-13,99)^{*}$ \\
\hline Consumo de alcohol & 7 & 22 & 10 & 75 & $2,38(0,81-7,00)$ \\
\hline Consumo de tabaco & 3 & 26 & 4 & 81 & $2,33(0,49-11,12)$ \\
\hline $\begin{array}{l}\text { Consumo de algún medicamento durante } \\
\text { el embarazo }\end{array}$ & 21 & 8 & 46 & 39 & $2,22(0,88-5,54)$ \\
\hline Uso de algún medicamento por vía vaginal & 9 & 20 & 22 & 63 & $1,2(0,5-3,2)$ \\
\hline Metonidazol vaginal & 31 & 7 & 67 & 18 & $1,2(0,45-3,37)$ \\
\hline Algún signo o síntoma durante el embarazo & 3 & 26 & 21 & 64 & $2,8(0,78-10,3)$ \\
\hline Sangrado vaginal durante el embarazo & 5 & 24 & 11 & 74 & $1,40(0,44-4,43)$ \\
\hline Vacuna toxoide tetánico & 22 & 6 & 53 & 26 & $2,10(0,77-5,76)$ \\
\hline Consumo de vitaminas & 21 & 8 & 72 & 13 & $1,79(0,65-4,97)$ \\
\hline Enfermedad aguda durante el embarazo & 19 & 10 & 46 & 39 & $1,61(0,67-3,87)$ \\
\hline Infección de vías urinarias durante el embarazo & 11 & 18 & 21 & 64 & $1,86(0,75-4,56)$ \\
\hline Vaginosis durante el embarazo & 9 & 20 & 29 & 56 & $0,86(0,35-2,14)$ \\
\hline
\end{tabular}

* Variables con diferencia estadísticamente significativa. 


\section{Discusión}

Se encontró una mayor frecuencia de casos en Cali, lo que podría relacionarse con un mayor número de nacimientos o con un mayor número de embarazos de alto riesgo remitidos a esta institución.

En cuanto a la frecuencia por subtipos de displasias esqueléticas, se encontró que en más de la mitad de los casos no se hizo un diagnóstico específico (clasificados como "otras osteocondrodisplasias") (tabla 3). La carencia de un diagnóstico preciso dificulta el adecuado abordaje clínico del paciente, así como el correcto asesoramiento a los padres sobre el pronóstico y la supervivencia[3]. Este hecho demuestra el pobre manejo que se da en nuestro medio a este grupo de enfermedades, tanto en el embarazo como en el periodo neonatal. Por esta razón, cabe resaltar la importancia de contar con un equipo multidisciplinario (compuesto por genética, radiología y neonatología), que se ocupe de abordar integralmente el caso y a sus familiares, desde el momento en que se tiene la sospecha diagnóstica en adelante, con el objetivo de repercutir en la morbilidad y mortalidad que producen estas alteraciones.

Posteriormente, en orden de frecuencia, se encontraron con el mismo número de casos la acondrodisplasia, la displasia tanatofórica y la anomalía por reducción de miembros. En último lugar aparecen la osteogénesis imperfecta y la displasia campomélica. Esta frecuencia de presentación de los diferentes tipos de displasias esqueléticas encontradas en este estudio concuerda con estudios previos[3].

En la búsqueda de casos asociados a displasias, se analizaron diferentes variables, tanto cuantitativas como cualitativas (tablas 4 y 5). Se encontró que, en el grupo de los casos, el número de gestaciones fue mayor que en el grupo de los controles, hecho que debe ser analizado con mayor precisión en futuros estudios para tratar de esclarecer si se trata de una asociación directa o secundaria a la mayor probabilidad de aparición del hecho.

Otra de las asociaciones de mayor importancia fue con la edad paterna de los casos, que superó, en promedio, siete años al promedio de la edad paterna de los controles; además, se encontró que el riesgo de displasias esqueléticas ya se encuentra aumentado cuando los padres tienen sus hijos a los 40 ó más años. En la literatura científica ya se ha reportado previamente a la edad paterna por encima de 55 años, como un factor de riesgo claramente establecido para el aumento en la frecuencia de displasias esqueléticas[1].

Otras asociaciones estadísticamente significativas que se presentaron fueron el bajo peso al nacer, el bajo peso para la edad de gestación y la prematuridad. Estos hallazgos se relacionan con 
situaciones fisiopatológicas propias de este grupo de enfermedades; si bien el principal sistema afectado es el esquelético, en muchos subtipos de displasias, existen alteraciones concomitantes en otros órganos y sistemas, que redundan en complicaciones durante el crecimiento y desarrollo fetales[2,3]. En este punto, es necesario resaltar el rol del médico que practica las ecografías prenatales, quien debe incluir a las displasias esqueléticas dentro de sus posibilidades diagnósticos, siempre que encuentre bajo peso fetal. En estudios anteriores se ha reportado que, ante este hallazgo, se debe buscar una correlación con la disminución en más de dos desviaciones estándar de los parámetros biométricos, para aumentar o descartar la sospecha diagnóstica[8].

No se encontraron asociaciones entre las displasias esqueléticas y el resto de variables medidas durante el embarazo (consumo de tabaco, alcohol, la presencia de signos y síntomas de enfermedades agudas). Tampoco se encontraron asociaciones con la exposición a los medicamentos más frecuentemente utilizados durante el embarazo (analgésicos, y antifúngicos vaginales y sistémicos), ni con actividades de inmunoprofilaxis y quimioprofilaxis durante el mismo periodo.

El estudio presentó limitaciones en cuanto al tamaño de la muestra, en parte por la baja frecuencia de presentación de este grupo de enfermedades, así como por el probable subregistro de las mismas. No fue posible analizar variables del periodo previo a la concepción, debido a la carencia de las mismas en la base de datos y a la dificultad de comunicación con las familias para obtener información adicional. Se sugiere la realización de nuevos estudios, para tratar de establecer factores de riesgo adicionales durante el periodo previo a la concepción.

\section{Conclusiones}

El diagnóstico temprano y preciso de las displasias esqueléticas redundará en una mejor evaluación del pronóstico del recién nacido y la asesoría de la pareja para calcular el riesgo de recurrencia en futuros embarazos. Esta es una enfermedad que impacta la morbimortalidad de nuestra población y es importante su conocimiento y manejo.

\section{Agradecimientos}

Este trabajo fue realizado con datos del Estudio Colaborativo Latinoamericano de Malformaciones Congénitas, ECLAMC.

Al Hospital Universitario San Ignacio, Fundación Emanuel, Clínica David Restrepo, Hospital Simón Bolívar, Hospital Universitario del Valle y Hospital Universitario de Caldas.

A los médicos que colaboraron con la recolección de la información. 


\section{Bibliografía}

1. Kumar P, Burton B. Congenital malformations: Evidence-based evaluation and management. First edition. Chicago: McGraw-Hill. 2008; 307-20.

2. Newman B, Wallis GA. Skeletal dysplasias caused by a disruption of skeletal patterning and endochondral ossification. Clin Genet. 2003;63:241-51.

3. Spranger JW, Brill PW, Poznanski AK. Bone dysplasias. In: Atlas of genetic disorders of skeletal development. Second edition. New York: Oxford University Press. 2002;9-14.

4. Hall CM. International nosology and classification of constitutional disorders of bone (2001). Am J Med Genet. 2002;113:65-77.
5. Krakow D, Lachman R, Rimoin D. Guidelines for the prenatal diagnosis of fetal skeletal dysplasias. Genet Med. 2009;11:127-33.

6. Rasmussen SA, Bieber FR, Benacerraf BR, Lachman RS, Rimoin DL, Holmes LB. Epidemiology of osteochondrodysplasias: Changing trends due to advances in prenatal diagnosis. Am $J$ Med Genet. 1996;61:49-58.

7. Zarante I, Franco L, López C, Fernández N. Frecuencia de malformaciones congénitas. Evaluación y pronóstico de 53.744 nacimientos en cuatro ciudades colombianas. Biomédica. 2010;30:1-23.

8. Schramm T, Gloning KP, Minderer S, Daumer-Haas C, Hörtnagel K, Nerlich $\mathrm{A}$, et al. Prenatal sonographic diagnosis of skeletal dysplasias. Ultrasound Obstet Gynecol. 2009;34:160-70. 